\title{
Recomendaciones generales en cirugía oncológica de cabeza y cuello durante la pandemia COVID-19
}

\author{
Angélica Silva-Figueroa ${ }^{1,2}$, Alejandra Gallego-Cifuentes ${ }^{2,3}$ y Marcelo Veloso-Olivares ${ }^{4}$
}

\section{General recommendations in head \& neck oncologic surgery during COVID-19 pandemic}

During the COVID-19 pandemic, the national and international health confrontation has focused its efforts on reducing the inherent risk of cancer patients. There is a strengthening in the resolutions of critical oncological cases that require early treatment, avoiding unnecessary exposure to SARS-CoV-2 in elective or deferrable cases. And also, risk-based safety considerations for head and neck surgeons consistent with the evidence available to date. These actions aim to avoid exposure to the virus and decrease the use of limited supplies, focusing our efforts on critical surgical treatment.

Key words: COVID-19; SARS-CoV-2; head \& neck surgery.

\section{Resumen}

Durante la pandemia COVID-19, el enfrentamiento sanitario nacional e internacional ha enfocado sus esfuerzos en disminuir los riesgos inherentes del paciente oncológico. Existe un reforzamiento del enfoque resolutivo en los casos oncológicos críticos que requieren tratamiento precoz, evitando la exposición innecesaria al SARS-CoV-2 en los casos electivos y/o diferibles. Así como también, consideraciones de seguridad según riesgo para los cirujanos de cabeza y cuello acorde con la evidencia disponible hasta la fecha. Estas medidas tienen por objetivo evitar la exposición al virus y disminuir el uso de insumos limitados, enfocando nuestros esfuerzos en el tratamiento quirúrgico crítico.

Palabras clave: COVID-19; SARS-CoV-2; cirugía cabeza y cuello.

\section{Introducción}

En contexto a los cambios nacionales con respecto a la contingencia en el ámbito sanitario, es importante que demostremos un alineamiento, más aún respecto a la experiencia demostrada en países desarrollados relacionado con el cuidado integral del paciente oncológico durante la pandemia COVID-19, ajustándola a nuestra realidad local. La escalada y dificultades para contener la infección por COVID-19 en el área de salud, hace que los centros de atención médica especializados entren en una presión asistencial acelerada y en aumento, forzándolos a enfocar sus esfuerzos asistenciales en identificar a los pacientes con COVID-19 y tratarlos, haciendo uso de equipos humanos, insumos y equipamiento médico previamente destinado a otras patologías, como es el cáncer. Y más aún, esfuerzos que también van orientados a evitar la transmisión cruzada nosocomial potencial a los pacientes con cáncer, que son parte del grupo de alto riesgo ${ }^{1}$. Países desarrollados en el área oncológica han decidido modificar la presente semana, sus guías de recomendación y manejo, basados en la experiencia asiática oncológica del reporte publicado recientemente en The Lancet Oncology por Dr. Wenhua Liang et al. ${ }^{2}$, que mostraron que los pacientes oncológicos poseen un riesgo mayor de infección por SARS-CoV-2 que la población general (odds ratio [OR], 2,31; 95\% $\mathrm{CI}, 1,89-3,02)^{2,3}$. Además, tienen un riesgo elevado de complicaciones respiratorias severas con manejo en unidades de cuidado intensivo, más aún en el subgrupo de pacientes con cáncer que tienen por antecedente una cirugía o quimioterapia efectuada en el mes previo a la infección COVID-19 (OR, 5,34; 95\% CI, 1,80-16,18; $\mathrm{p}=0,0026)^{2}$.
'Facultad de Medicina Departamento de Cirugía, Universidad Finis Terrae. Santiago, Chile.

${ }^{2}$ Servicio de Cirugía,

Departamento de Cirugía de Cabeza y Cuello, Hospital Barros Luco-Trudeau. Santiago, Chile. ${ }^{3}$ Servicio de Cirugía Departamento de Cirugía Oncológica, Hospital Militar de Santiago. Santiago, Chile. ${ }^{4}$ Instituto Nacional del Cáncer, Departamento de Cirugía de Cabeza y Cuello. Santiago, Chile.

Recibido el 18 de abril de 2020 y aceptado para publicación el 23 de abril de 2020.

Correspondencia a: Dra. Angélica Silva F. angelica.silva@uft.cl 
En ese contexto, una demostración nacional de la validación de estas experiencias internacionales son las recomendaciones de la Sociedad de Cirujanos de $\mathrm{Chile}^{4}$, y las recomendaciones actuales del Ministerio de Salud, Decreto $\mathrm{N}^{\mathrm{o}}$ 11, Santiago 30 de marzo de $2020^{5}$, que delimita sus atenciones a cirugías de urgencia/emergencias, mantiene 18 patologías GES exigible para ser resueltas y favorece las intervenciones terapéuticas oncológicas más críticas, que podrían poner en detrimento el estado biomédico de un individuo en menos de $1 \mathrm{mes}^{4,5}$. Otras medidas que intentan resguardar la integridad de los pacientes oncológicos en los centros de salud son; favorecer el manejo ambulatorio, diferir los controles presenciales en la fase 4 , y educar sobre las reglas de protección para prevenir la transmisión en el hogar y en el ambiente quirúrgico.

En general todas estas medidas, se han ido modificando rápida y progresivamente según la evidencia disponible hasta la fecha. Hemos querido unificar los criterios de priorización recientemente publicados con respecto a cirugía oncológica de cabeza y cuello para favorecer la homogeneidad en el manejo, y además revisar las guías más actualizadas sobre el uso de las medidas de protección individual en el ámbito nosocomial en el área de la cirugía de cabeza y cuello.

\section{Procedimientos quirúrgicos}

Actualmente, se recomienda restringir los tratamientos quirúrgicos no críticos en cirugía, cabeza y cuello, focalizando la resolución de pacientes oncológicos locorregionalmente avanzados o cuyo retraso mayor a 30 días en el manejo implicaría un efecto deletéreo en su estado biomédico y, por lo tanto, no existe tratamiento equivalente y no puede ser diferido. Para ello hemos revisado y adaptado las recomendaciones de sociedades quirúrgicas tanto nacionales como internacionales disponibles en la literatura en cirugía cabeza y cuello ${ }^{6-10}$.

\section{Cirugía endocrina}

a. Las operaciones por enfermedades endocrinas no complicadas pueden ser retrasadas. Las enfermedades y presentación que pudieran calificar para cirugía urgente (es decir, dentro de 4-8 semanas durante la pandemia), incluye:

\section{Tiroides}

- Cáncer de tiroides que, actualmente, amenacen la vida, como; amenaza de morbilidad con invasión local (ej. tráquea, nervio laríngeo recurrente), biología agresiva (rápido crecimiento o recurrencia, cáncer medular, enfermedad locorregional rápidamente progresiva), y evidencia de enfermedad recurrente regional invasiva en imágenes y con histopatología compatible neoplásica.

- Biopsia abierta con intento diagnóstico de cáncer anaplásico sospechoso o linfoma.

- Enfermedades endocrinas no oncológicas que pudieran requerir resolución precoz; enfermedad de Basedow Graves severamente sintomática que ha fallado a tratamiento médico, bocio altamente sintomático o con riesgo de obstrucción de vía aérea.

b. En congruencia a MINSAL con Decreto $\mathrm{N}^{\mathrm{o}}$ 11, cito textual: "b. la suspensión en todo el territorio nacional, la obligatoriedad para el Fondo Nacional de Salud y las instituciones de Salud Previsional del cumplimiento de la garantía explícita de oportunidad por el plazo de un mes a contar del 30 de marzo de 2020" y "c. se excluyen de la presenta suspensión, todas las prestaciones cuya postergación y/o inejecución, de acuerdo con la etapa sanitaria en curso implique riesgo vital para el paciente, lo que será definido por el médico tratante"5. Exceptúa de esta suspensión a 18 patologías AUGE, incluyendo a linfoma. En este último, en presencia de adenopatías cervicales clínicas documentadas, requerirán de la programación de una biopsia incisional o excisional quirúrgica bajo anestesia general.

Con respecto al estudio diagnóstico en linfoma en adultos (linfoma B, linfoma Hodgkin), la biopsia quirúrgica es de primera elección debido a su mayor capacidad diagnóstica, aunque con morbilidad asociada. En la última década existe una tendencia acrecentada a recomendar en casos seleccionados, una biopsia core con anestesia local realizada por radiólogos en el ámbito ambulatorio. Esta alcanza un $65-75 \%$ de diagnóstico definitivo, con una menor morbilidad y una mayor aceptación fisiológica en pacientes con comorbilidades o adultos mayores que requieren pases preoperatorios. Incluso, en algunos casos, una punción con aguja fina/biopsia core en combinación con inmunohistoquímica $\mathrm{y}$ citrometría de flujo pudiera ser usado como primer intento diagnóstico según las recomendaciones 2020 del National Comprehensive Cancer Network para estudio de linfoma, y la revisión sistémica liderada por Frederiksen et al., en $2015^{11-13}$.

\section{Paratiroides}

- Hiperparatiroidismo con hipercalcemia incontrolable médicamente y severa con amenaza vital (generalmente estos casos están hospitalizados; 
completando su estudio con ecografía cervical, cintigrama paratiroides, exámenes preoperatorios y según comorbilidades evaluación preanestésica). Mantener alto nivel de sospecha de cáncer de paratiroides.

\section{Cirugía oncológica de cabeza y cuello no endocrina}

En congruencia a las recomendaciones nacionales e internacionales cada resolución de pacientes oncológicos debiese sopesarse según los múltiples factores asociados por el equipo tratante y el paciente; incluyendo comorbilidades, necesidad de camas críticas, riesgo quirúrgico asociado y pronóstico según el estadiaje oncológico ${ }^{4,6,10}$.

En caso de indicarse un tratamiento quirúrgico este debe enmarcarse en un manejo multidisciplinario oncológico y coordinado, en los que la oportunidad de recibir él o los tratamientos adyuvantes estén disponibles en los plazos adecuados en los que su efectividad sea la mejor para lograr el control de la enfermedad. Esto es crucial en el enfrentamiento del paciente oncológico en todo momento, no sólo dentro de esta pandemia, es por ello por lo que todos los pacientes deben ser presentados en un comité oncológico, idealmente vía teleconferencia, en el que todos los especialistas estén en conocimiento del paciente y su plan de tratamiento a seguir, para evitar así retrasos en la aplicación de tratamientos adyuvantes, lo que ensombrece el pronóstico de la enfermedad, haciendo que el esfuerzo quirúrgico realizado no cumpla su objetivo.

Las neoplasias malignas del tracto aerodigestivo superior son un desafío durante la pandemia COVID-19, teniendo en cuenta que la replicación viral de COVID-19 se anida en la cavidad nasal, nasofaringe y orofaringe, que son zonas rutinarias de manejo del equipo de cirugía cabeza y cuello tanto para evaluación clínica, procedimientos quirúrgicos de cirugía de cabeza y cuello (ej.; traqueostomías) y tratamientos quirúrgicos oncológicos. Los aerosoles liberados durante estos procedimientos potencialmente pudieran incrementar las probabilidades de infección para los profesionales de la salud.

Estableciendo las prioridades de atención podríamos considerar a aquellas neoplasias que requieran resolución terapéutica antes de 6 semanas, y que alcancen a tener un estudio completo de etapificación, exámenes y evaluaciones preoperatorias por otras especialidades según necesidad. Entre estos podemos considerar los cánceres poco o pobremente diferenciados, el cáncer escamoso de vía aéreodigestiva superior de alto riesgo, el melanoma maligno y los sarcomas de alto grado entre otros.

\section{Cáncer escamosos de cabeza y cuello}

- En la medida de lo posible, considerar privilegiar la resección oncológica por sobre reconstrucciones complejas, y favorecer colgajos locales $\mathrm{u} / \mathrm{o}$ regionales. Incluso, evaluar reconstrucciones en segundo tiempo si es posible.

- En general, el ideal es considerar casos que no requieran cama UTI o UCI, y de rápido recambio hospitalario, evitando en la medida de lo posible hospitalizaciones prolongadas (outliners $>7$ días). Aún cuando estas últimas recomendaciones, son difíciles de lograr en algunos casos escamosos de cabeza y cuello, debido a la envergadura de la cirugía efectuada y las comorbilidades asociadas de los pacientes.

Sarcomas de partes blandas de cabeza y cuello

- Sarcomas primarios de partes blandas de cabeza y cuello sin metástasis a distancia en su estudio de etapificación preoperatoria tendrían que ser priorizado para cirugía.

- Tumores lipomatosos atípicos de reciente diagnóstico, dermatofibrosarcomas protuberantes clásicos sin degeneración fibrosarcomatosa y desmoides pudieran ser diferidos por 3 meses

- Resección de sarcomas de bajo grado con conocido comportamiento biológico indolente y bajo riesgo metastásico (ej. liposarcoma mixoide, tumor fibromixoide de bajo grado) pudieran ser diferidos por 1 mes.

- Si hay indicación de radioterapia adyuvante, planear realizarla preoperatoria en casos seleccionados, principalmente, ya que esto puede ser administrado de forma ambulatoria y retrasaría la cirugía por 3-4 meses.

- Si hay indicación de quimioterapia neoadyuvante por sarcomas de alto grado o por enfermedad recurrente, considerar realizarla ambulatoriamente.

\section{Melanoma maligno cutáneo}

- Priorizar manejo quirúrgico de melanomas malignos según etapificación con biopsia diagnóstica documentada; T4/T3 ( $<2$ mm Breslow), T2/T1 $(<2 \mathrm{~mm})$, y finalmente, melanoma in situ.

- Biopsia linfonodo centinela en $>1 \mathrm{~mm}$ de Breslow, dentro de 3 meses del diagnóstico.

- Manejo clínico en Etapa III con terapia sistémica neoadyuvante. Si el paciente no es idóneo para este manejo según comité oncológico (no presencial), considerar resección quirúrgica. 


\section{Traqueostomía}

Del importante número de pacientes infectados con SARS-CoV-2, aproximadamente el 2,3 al $15,2 \%$ requerirán manejo en cuidados intensivos con ventilación mecánica $(\mathrm{VM})$ o sistema de oxigenación por membrana extracorpórea (ECMO) ${ }^{14-16}$. Por lo tanto, en los casos sometidos a VM prolongada pudieran requerir traqueostomía para optimizar el soporte ventilatorio. Existe una sola revisión de la literatura publicada por Tay et al, ${ }^{17}$, en Singapur, con respecto al uso de traqueostomía y síndrome respiratorio agudo severo (SARS), a nuestro conocimiento. En esta revisión se hace hincapié a los siguientes factores, que enumeraremos a continuación;

1. Las precauciones de barrera (EPP) son críticas y esenciales, por lo tanto, deben estar disponibles en cada uno de estos procedimientos. Incluso en algunos reportes se recomienda reforzar las barreras faciales con el uso de máscaras faciales completas herméticas con purificadores de aire (PAPRs). Teniendo en consideración que durante la epidemia SARS CoV-1 2003, estas barreras reforzadas mantuvieron a los equipos quirúrgicos libres de contagio durante toda la epidemia en las 23 traqueostomías quirúrgicas reportadas.

2. Otro factor importante, es que las traqueostomías quirúrgicas realizadas ya sea en el lecho de cama UCI o en pabellones quirúrgicos fueron indicadas con rigurosidad y planificadas meticulosamente. Dependiendo del escenario las consideraciones pudieran cambiar, pero en todas debiera realizarse con presión negativa, marcaciones bien delimitadas de espacio favoreciendo un área anterior separada de la zona potencialmente contaminada (doble puerta) y rutas de transportes o circulación bien señalizadas tanto dentro de la UCI como en pabellones.

3. Durante el procedimiento debe minimizarse al máximo la exposición a secreciones aerosolizadas, incluida sangre. Por lo tanto, el equipo completo debe estar entrenado para asegurar que, el paciente no tosa, detener VM justo antes de realizar traqueotomía y idealmente no usar succión. Todo este procedimiento al igual que todo procedimiento de alto riesgo debe ser realizado con sistema cerrado con filtro vírico.

4. Por otro lado, la experiencia del equipo es relevante para minimizar los tiempos y riesgos. Es por ello, que en los reportes la mayoría de las traqueostomías quirúrgicas eran realizadas habitualmente por un equipo (cirujano, anestesista, enfermera y técnicos) previamente familiarizados con la técnica y con parámetros preestablecidos rutinariamente, debido a la dificultad de conver- sación durante el procedimiento por el uso de EPP y PAPRs. Además, un factor en común, es la limitación del número de participantes en cada procedimiento: a un cirujano, un ayudante quirúrgico experimentado, un especialista de cuidados intensivo, una enfermera quirúrgica o arsenalera experimentada y un anestesista. Cada uno de los participantes regulares a este procedimiento es recomendable que sean monitorizados tanto en temperatura y en sintomatología respiratoria antes y después del procedimiento, con un seguimiento estricto epidemiológico estricto de parte de la institución. Debido a que este procedimiento es uno de los de mayor riesgo de contagio en el área nosocomial.

5. Luego de realizado el procedimiento es de extrema relevancia que el manejo del equipo contaminado sea realizado con las más estrictas medidas de descontaminación y desecho en doble bolsa. $\mathrm{Y}$ el personal encargado de este proceso también debe estar protegidos con medidas EPP. Todos los instrumentos usados deben ser colocados en bolsas biodegradables y luego, dentro de otra bolsa de desechos con riesgos biológicos para ser transportada y llevada a los centros de esterilización ${ }^{18}$.

Cabe agregar, que la traqueostomía percutánea requiere mayor manipulación de la vía aérea, lo que aumentaría el riesgo de aerosolización al compararlo con la traqueostomía quirúrgica, es por ello, que durante la epidemia SARS-CoV-1 se favoreció el uso de la quirúrgica ${ }^{17}$. Aunque en la última década ha habido grandes avances en la técnica percutánea, agregado a un mayor nivel de experticia en su ejecución. Desafortunadamente, por ahora, no han sido reportadas experiencias en la literatura durante la pandemia COVID-19, y aún es debatible su documentada capacidad de generación de aerosoles, debido a que la evidencia es muy limitada.

\section{Consideraciones de seguridad para cirujanos que realizamos cirugías de cabeza y cuello}

Existen 3 factores a considerar para el uso de medidas de protección física para cirujanos cabeza y cuello durante la contingencia COVID-19; primero, previamente explicado, el nivel de replicación viral es alta en zona aereodigestivas altas, por lo tanto, cualquier exposición directa a esta zona durante un examen físico bucal o cirugía que exponga estas zonas directamente en un paciente sintomático o 
no sintomático, aumenta el riesgo de infección y además, según el número y tiempo de exposición aumenta la carga viral en el trabajador de salud ${ }^{19}$. Segundo, la transmisión de SARS-CoV-2 sabemos que no solo es a través de individuos sintomáticos, sino también por individuos SARS-CoV-2 positivos asintomáticos (SARS-Cov-2+A) y por objetos inanimados. Con respecto a los pacientes SARS-CoV-2+A debido a los reportes asiáticos con test PCR de aplicabilidad masiva, se identificó una incidencia del $25 \%-30 \%{ }^{20,21}$, que, por lo tanto, no son identificados en los sistemas de triage existentes ni por evaluaciones clínicas y ni siquiera por el propio individuo portador y que, además, su capacidad de transmisibilidad alcanza su peak a los 2,68-2,88 días previo al desarrollo de síntomas ${ }^{21-23}$. Y tercero, se suma la reportada mayor estabilidad en superficies inertes de este virus SARS-CoV-2 en comparación con SARS-CoV-1, logrando que su transmisibilidad en ambientes nosocomiales sea de un manejo aún más complejo entre paciente y paciente, y, por lo tanto, se recomienda asumir de que la sala de operaciones estará contaminada, y requerirá un aseo terminal y acucioso ${ }^{24,25}$. Todo lo anterior hace que los trabajadores de la salud sean considerados de alto riesgo alcanzando una tasa de COVID-19 positivo del 3,8\% al 10\% en reportes de China, Italia y España, principalmente en aquellas especialidades destinadas directamente al cuidado de los pacientes COVID-1926. Además, el reporte preliminar recientemente publicado por Ing et al. ${ }^{27}$, destaca una frecuencia de mortalidad según especialidad de urgenciólogo 40,6\%, Medicina 5,8\% y en tercer lugar otorrinolaringología/cirugía cabeza y cuello con 4,2\%. Es por ello, que las medidas de protección individual son consideradas exigibles para mantener la seguridad de los profesionales de la salud, según la actividad de salud que realicen.

En esta tabla resumimos y adaptamos las recomendaciones de equipo de protección individual en cirugía cabeza y cuello según la evidencia actualmente disponible durante la contingencia epidemiológica de COVID-19 y según el riesgo de transmisibilidad de SARS-CoV-2 al equipo médico durante la atención en salud ${ }^{17,28,29}$ (Tabla 1).

En particular, en las cirugías oncológicas en las cuales se exponga la vía aérea o la mucosa orofaríngea, se recomienda evitar el uso de sistema de electrocoagulación eléctrica, ultrasónicos, microdebridadores, fresas, sierras quirúrgicas o cualquier sistema que pueda generar aerosoles particulados. $\mathrm{Y}$ en caso de que sea estrictamente necesario, reducir al máximo el tiempo de exposición, debido al riesgo potencial de aerosolización de sangre infectada con SARS-CoV-2 ${ }^{10,25}$. Además, es importante que los sistemas de aspiración sean a circuito cerrado con filtro antivírico e idealmente a presión negativo independiente del nivel de sospecha de COVID-19.

Estas recomendaciones están sujetas a modificaciones según la información disponible que se vaya reportando en la literatura internacional. Pero es importante tomar en consideración que estos insumos estén disponibles en los servicios de cirugía y en pabellones, según evaluación clínica y/o procedimientos quirúrgicos que realice cada equipo de cirugía de cabeza y cuello.

\section{Seguimiento de pacientes con cirugía diferida}

Es importante también que durante la contingencia haya una coordinación entre los departamentos multidisciplinares facilitando el seguimiento clínico de estos casos que, actualmente, no son prioridad critica, Ej.: radiología, laboratorio.

Tiroides:

- Ecografía cervical (incluyendo linfonodos cervicales del I a VI bilaterales) + examen físico cada 3 a 6 meses.

- Hormonas tiroideas.

- Marcadores oncológicos; tiroglobulina, anticuerpos tiroglobulina, calcitonina y antígeno carcinoembrionario.

Paratiroides:

- Manejo médico por endocrinología y seguimiento clínico.

Además de implementar seguimiento oncológico telefónicos o vía telemedicina en los centros que tengan habilitado este recurso, mas considerando el reporte retrospectivo en pacientes con cáncer en China que mostró que las visitas hospitalarias repetidas y hospitalizaciones son potenciales factores de riesgo para COVID-19 $9^{30}$.

\section{Conclusiones}

En estos momentos de la contingencia epidemiológica COVID-19, la primordial misión de los centros de salud enfocados en el tratamiento de pacientes con cáncer de cabeza y cuello es la protección del bienestar de sus pacientes y sus profesionales. Existen varias medidas reportadas tanto nacionales 
CIRUGÍA AL díA

Tabla 1.

\begin{tabular}{|c|c|c|}
\hline Riesgo y definición & Pacientes & Staff/residentes \\
\hline \multicolumn{3}{|c|}{ Pacientes no inmunocomprometidos evaluados sin procedimiento quirúrgico (examen físico) } \\
\hline $\begin{array}{l}\text { Alto riesgo para staff/residentes: } \\
\text { Paciente con COVID-19 + activo } \\
\text { Paciente con sintomas de influenza } \\
\text { Paciente con COVID-19 en sospecha }\end{array}$ & Mascarilla quirúrgica & $\begin{array}{l}\text { Mascarilla N95 } \\
\text { Protector ocular o protector facial completo } \\
\text { Pechera completa con mangas largas } \\
\text { Guantes }\end{array}$ \\
\hline $\begin{array}{l}\text { Bajo riesgo para staff/residentes: } \\
\text { Otro examen fisico en paciente asintomático }\end{array}$ & Mascarilla & $\begin{array}{l}\text { Mascarilla } \\
\text { Guantes }\end{array}$ \\
\hline \multicolumn{3}{|c|}{$\begin{array}{l}\text { Procedimiento de riesgo de generación de aerosoles (cirugía, traqueostomía, manejo } \\
\text { de traqueostomía, instrumentación de mucosa de cabeza y cuello, nasofaringolaringoscopia) }\end{array}$} \\
\hline $\begin{array}{l}\text { Bajo riesgo: } \\
\text { Pacientes asintomáticos y no testeados o test } \\
\text { negativo en } 48 \text { horas previo al procedimiento }\end{array}$ & Nada* & $\begin{array}{l}\text { Mascarilla N95, y protector ocular (usar } \\
\text { protector facial completo para reusar N95) } \\
\text { Pechera completa con mangas largas } \\
\text { Doble guantes }\end{array}$ \\
\hline \multicolumn{3}{|c|}{ *Si es posible, testee a los pacientes dentro de $48 \mathrm{~h}$ previas al procedimiento. } \\
\hline \multicolumn{3}{|c|}{$\begin{array}{l}\text { Procedimiento sin riesgo de generación de aerosoles (cirugía de tejidos blandos superficial, } \\
\text { esto pudiera tener cierta carga viral el riesgo es más bajo, a menos que la sangre sea aerolizada) }\end{array}$} \\
\hline
\end{tabular}

Abreviaciones: COVID-19, infección por coronavirus-2; COVID-19+ activo, infección por coronavirus-2 con test positivo y sintomático. Adaptado al español, Givi et $\mathrm{al}^{28}$. Safety Recommendations for Evaluation and Surgery of the Head and Neck During the COVID-19 Pandemic, JAMA. *Se debe tomar en consideración, las actuales recomendaciones nacionales chilenas en alineación con las recomendaciones del Center for Disease Control and Prevention (CDC), desde abril 3, 2020; "todo individuo que circule en ambientes públicos debieran usar una máscara facial, que pudiera ser no quirúrgica".

como internacionales, que tienen por finalidad proveer al paciente del mejor cuidado disponible y de la forma más segura.

En general una de las formas más efectivas para evitar la exposición al virus y disminuir el uso de insumos limitados hospitalarios, es evitando proce- dimientos quirúrgicos no críticos, como también lo recomendó The American College of Surgeons, recientemente $^{31}$. De esta manera reducir el número de contagios tanto en los usuarios como en el personal a nivel nosocomial, preservar el equipamiento de protección individual y así, limitar y mitigar la con- 
gestión incontenible que se evidenció en los países desarrollados.

Todos estamos aprendiendo al paso progresivo y rápido de esta pandemia, y con la información actualmente disponible, esperamos mantener la salud de nuestra comunidad usuaria y quirúrgica e ir adaptándonos a la nueva evidencia disponible que nos permita mantener el cuidado efectivo, integral y de bajo riesgo, cuando nuestros pacientes oncológicos de cabeza y cuello lo requieran.

\section{Responsabilidades éticas}

Protección de personas y animales. Los autores declaran que para esta investigación no se han realizado experimentos en seres humanos ni en animales.

Confidencialidad de los datos. Los autores declaran que en este artículo no aparecen datos de pacientes.

Conflictos de interés: no hay.

\section{Bibliografía}

1. The Lancet O. COVID-19: global consequences for oncology. Lancet Oncol. 2020;21(4):467. DOI: 10.1016/S14702045(20)30175-3

2. Liang W, Guan W, Chen R, Wang W, Li $\mathrm{J}, \mathrm{Xu} \mathrm{K}$, et al. Cancer patients in SARS$\mathrm{CoV}-2$ infection: a nationwide analysis in China. Lancet Oncol. 2020;21:335-7. DOI: 10.1016/S1470-2045(20)30096-6

3. You B, Ravaud A, Canivet A, Ganem G, Giraud P, Guimbaud R, et al. The official French guidelines to protect patients with cancer against SARS-CoV-2 infection. Lancet Oncol. 2020. DOI: 10.1016/S14702045(20)30204-7

4. Sociedad de Cirujanos de Chile. Recomendaciones de la Sociedad de Cirujanos de Chile para cirugía en pacientes COVID-19, 2020: Sociedad de Cirujanos de Chile; 2020 [Available from: http://www.socich.cl/2020/03/29/ recomendaciones-para-cirugia-enpacientes-covid-19/].

5. República de Chile MINSAL. SUSPENDE GARANTÍA DE OPORTUNIDADES DE LAS GARANTIÍAS EXPLÍCITAS EN SALUD EN LOS PROBLEMAS DE SALUD QUE INDICA. DECRETO N ${ }^{\circ} 11$ MINSAL (30 MAR 2020). Diario Oficial; 2020.

6. Society of Surgical Oncology. Cancer Surgeries in the Time of COVID-19: A Message from the SSO President and President-Elect, 2020: Society of Surgical Oncology; 2020 [Available from: https://www.surgonc.org/wp-content/ uploads/2020/03/COVID-19-Letter-toMembers.pdf].

7. Society of Surgical Oncology. Resource for Management Options of Endocrine/ Head and Neck Cancer During COVID-19, 2020: Society of Surgical Oncology (SSO); 2020 [Available from: https://www.surgonc.org/wpcontent/uploads/2020/03/EndocrineHead-and-Neck-Resource-duringCOVID-19-3.30.20.pdf].

8. Society of Surgical Oncology. Resource for Management Options of Sarcoma During COVID-19, 2020: Society of Surgical Oncology (SSO); 2020 [Available from: https://www.surgonc.org/ wp-content/uploads/2020/03/SarcomaResource-during-COVID-19-3.30.20.pdf].

9. Society of Surgical Oncology. Resource for Management Options of Melanoma During COVID-19, 2020: Society of Surgical Oncology (SSO); 2020 [Available from: https://www.surgonc.org/ wp-content/uploads/2020/03/MelanomaResource-during-COVID-19-3.30.20.pdf].

10. Sociedad Española de

Otorrinolaringología y Cirugía de Cabeza y Cuello. Recomendaciones De La Seorl-Ccc Para La Práctica De La Especialidad Durante La Pandemia De Covid-19, 2020: Sociedad Española de Otorrinolaringología y Cirugía de Cabeza y Cuello (SEORL); 2020 [Available from: https://seorl. net/wp-content/uploads/2020/03/ Recomendaciones-de-la-SEORL-CCC-22de-marzo-de-2020.pdf].

11. Frederiksen JK, Sharma M, Casulo C, Burack WR. Systematic review of the effectiveness of fine-needle aspiration and/ or core needle biopsy for subclassifying lymphoma. Arch Pathol Lab Med. 2015;139:245-51. DOI: 10.5858 arpa.2013-0674-RA.

12. National Comprehensive Cancer Network.
NCCN Clinical Practice Guidelines in Oncology: B-Cell Lymphomas 2020. Available from: https://www.nccn.org/ professionals/physician_gls/pdf/b-cell.pdf.

13. National Comprehensive Cancer Network NCCN Clinical Practice Guidelines in Oncology: Hodgkin Lymphoma 2020. Available from: https://www.nccn.org/ professionals/physician_gls/pdf/hodgkins. pdf.

14. Guan WJ, Ni ZY, Hu Y, Liang WH, Ou $\mathrm{CQ}, \mathrm{He} \mathrm{JX}$, et al. Clinical Characteristics of Coronavirus Disease 2019 in China. N Engl J Med. 2020. DOI: 10.1056/ NEJMoa2002032.

15. Huang C, Wang Y, Li X, Ren L, Zhao J, Hu Y, et al. Clinical features of patients infected with 2019 novel coronavirus in Wuhan, China. Lancet. 2020;395(10223):497-506. DOI: 10.1016/ S0140-6736(20)30183-5.

16. Zhou F, Yu T, Du R, Fan G, Liu Y, Liu Z, et al. Clinical course and risk factors for mortality of adult inpatients with COVID-19 in Wuhan, China: a retrospective cohort study. Lancet. 2020;395(10229):1054-62. DOI: 10.1016/ S0140-6736(20)30566-3.

17. Tay JK, Khoo ML, Loh WS. Surgical Considerations for Tracheostomy During the COVID-19 Pandemic: Lessons Learned From the Severe Acute Respiratory Syndrome Outbreak. JAMA Otolaryngol Head Neck Surg. 2020. DOI: 10.1001/jamaoto.2020.0764.

18. Chee VW, Khoo ML, Lee SF, Lai YC, Chin NM. Infection control measures for operative procedures in severe acute respiratory syndrome-related patients. Anesthesiology. 2004;100:1394-8. DOI: 10.1097/00000542-20040600000010 . 
19. Zou L, Ruan F, Huang M, Liang L, Huang H, Hong Z, et al. SARS-CoV-2 Viral Load in Upper Respiratory Specimens of Infected Patients. N Engl J Med. 2020;382(12):1177-9. DOI: $10.1056 / \mathrm{NEJMc} 2001737$.

20. Zhang GM, Luo L, Ding XM, Dong DH, Li B, Ma XC, et al. MicroRNA-126 inhibits tumor cell invasion and metastasis by downregulating ROCK1 in renal cell carcinoma. Mol Med Rep. 2016;13:50292036. DOI: $10.1007 / \mathrm{s} 11684-020-0766-9$.

21. Bai Y, Yao L, Wei T, Tian F, Jin DY, Chen L, et al. Presumed Asymptomatic Carrier Transmission of COVID-19. JAMA. 2020. DOI: $10.1001 /$ jama.2020.2565.

22. WE W, Z L, CJ C, SE Y, MP T, VJ L. Presymptomatic Transmission of SARSCoV-2, Singapore, January 23, March 16, 2020. MMWR Morb Mortal Wkly Rep. 2020. DOI: http://dx.doi.org/10.15585/ mmwr.mm6914e1.

23. Rothe C, Schunk M, Sothmann P, Bretzel G, Froeschl G, Wallrauch C, et al. Transmission of 2019-nCoV Infection from an Asymptomatic Contact in Germany. N Engl J Med. 2020;382:970-1.
DOI: 10.1056/NEJMc2001468.

24. van Doremalen N, Bushmaker T, Morris DH, Holbrook MG, Gamble A, Williamson BN, et al. Aerosol and Surface Stability of SARS-CoV-2 as Compared with SARS-CoV-1. N Engl J Med. 2020. DOI: 10.1056/NEJMc2004973.

25. Brat GA, Hersey SP, Chhabra K, Gupta A, Scott J. Protecting Surgical Teams During the COVID-19 Outbreak: A Narrative Review and Clinical Considerations. Annals of Surgery. 2020. https://journals. lww.com/annalsofsurgery/Documents/ COVID\%20Surgery_VF.pdf.

26. Cinar P, Kubal T, Freifeld A, Mishra A, Shulman L, Bachman J, et al. Safety at the Time of the COVID-19 Pandemic: How to Keep our Oncology Patients and Healthcare Workers Safe. NCCN Best Practices Committee. 2020. DOI: 10.6004/ jncen.2020.7572.

27. Ing EB, Xu A, Salimi A, Torun N. Physician Deaths from Corona Virus Disease (COVID-19). The Preprint Server for Health Sciences medRxiv. 2020. DOI: https://doi.org/10.1101/2020.04.05.2 0054494 .

28. Givi B, Schiff BA, Chinn SB, Clayburgh
D, Iyer NG, Jalisi S, et al. Safety Recommendations for Evaluation and Surgery of the Head and Neck During the COVID-19 Pandemic. JAMA Otolaryngol Head Neck Surg. 2020. DOI: 10.1001/ jamaoto.2020.0780.

29. Centers of Disease Control and Prevention. Strategies to Optimize the Supply of PPE and Equipment for Healthcare Professionals: Centers of Disease Control and Prevention; 2020 [Available from: https://www.cdc.gov/ coronavirus/2019-ncov/hcp/ppe-strategy/ index.html.

30. Yu J, Ouyang W, Chua MLK, Xie C. SARS-CoV-2 Transmission in Patients With Cancer at a Tertiary Care Hospital in Wuhan, China. JAMA Oncol. 2020. DOI: 10.1001/ jamaoncol.2020.0980.

31. American College of Surgeons. COVID 19: Elective Case Triage Guidelines for Surgical Care Updated March 27, 2020: American College of Surgeons; 2020 [Available from: https://www.facs.org/-/ media/files/covid19/guidance_for_triage of_nonemergent_surgical_procedures. ashx.

*Este documento ha sido elaborado y revisado por socios y miembros del directorio de la Sociedad Chilena de Cirugía Cabeza, Cuello y Plástica Maxilofacial, como recomendaciones en la atención quirúrgica de pacientes oncológicos de cirugía cabeza y cuello, durante la pandemia COVID-19. 